\title{
THEORIE UND PRAXIS
}

\section{$E \rrbracket N E \quad$ UMFRG \\ UN $\mathbb{R} \mathbb{R}$ \\ $S T \cup D \| E R E N D E N \quad \mathbb{Z} \cup \mathbb{M}$ \\ LANDESKUNDEUNTERRICHT

L andeskunde war lange ein Stiefkind innerhalb des spanischen Deutschunterrichts und Sprachstudiums. In den letzten Jahren findet allerdings mit zunehmender Reflexion über Lernmethoden

\section{Elisabeth F. Basteck}

"Landeskunde lässt sich definieren als die Gesamtheit aller Informationen und Deutungstheoreme, die dazu die-

Lehrtechniken auch hier ein

Umdenken statt: weg vom lehrerzentrierten Frontaldozieren historischer Epochen hin zu interkulturellen Kommunikationsübungen und kontrastivem Kulturtraining.' Für die Studiengänge Deutsche Philologie und Übersetzungswissenschaften an den spanischen Universitäten gilt allerdings immer noch die Vorgabe seitens des MEC: „Historia y cultura alemana. Aspectos geograficos, históricos, ártisticos y culturales", d.h. z.B. für die Germanistik ein eigenständiges Fach "Historia y cultura alemana" ${ }^{\prime 2}$

Dieses Konzept von Landeskunde / cultura y sociedad (oder ähnlich lautenden Seminartiteln an den Universitäten) steht in der Tradition der "lenguas clásicas", nach deren Vorbild die "lenguas vivas" seit dem Gesetz "Ley Pidal" 1845 in das öffentliche Bildungssystem in Spanien aufgenommen wurden ${ }^{3}$. Bis heute orientiert sich das staatlichvorgeschriebene Lehrprogramm am traditionellen, bildungsbürgerlichen Verständnis von Kultur: d.h. Landeskunde $=$ Wissen, mit dem man sich als individuelle Persönlichkeit bildet, um sich anschließend als "gebildete Persönlichkeit" in höheren Gesellschaftskreisen darstellen zu können:

Dies hat nichts mit den heute üblichen Landeskundekonzepten zu tun. So heißt es im internationalen Handbuch "Deutsch als Fremdsprache" (2001), nen, das Interaktionswissen (z.B. Begrüßungsformeln, Wahrnehmungs- und Mentalitätsunterschiede) eines jeweiligen Sprachlerners zu optimieren, sein Verständnis der Zielkultur und ihrer historischen und gesellschaftlichen Bedingungen zu verbessern und ihn darüber hinaus in die Lage zu versetzen, sich der verschiedenen Mechanismen der fremdkulturellen Lern- und Wahrnehmungsprozesse bewusst zu werden. ${ }^{\prime 4}$

Penning (1995) differenziert den Lehrauftrag für den Landeskundeuntericht innerhalb der Fremdsprachenlehre weiter:

"Landeskunde ist die Beschäftigung mit den primär nichtsprachlichen Bedingungen, Regelwerken und präsupponierten Wissensbeständen von Kommunikation, um in einer fremden Kultur kommunikative Zusammenhänge richtig zu deuten und sich selbst in einem Prozess des vorsichtigen "Aushandelns" angemessen zu verhalten. ${ }^{15}$

Pennings Einteilung der Lehrinhalte ist daher in 4 Bereiche untergliedert:

1. sprachbezogene, implizite Landeskunde

2. anthropologische Landeskunde

3. explizite, programmatische Landeskunde

4. landesübergreifende Problematisierung

Die landeskundlichen Lehrinhalte ordnet er wie folgt: 


\begin{tabular}{|l|l|l|l|l|}
\cline { 2 - 4 } $\begin{array}{c}\text { Sprachbezogene, } \\
\text { implizite Landeskunde }\end{array}$ & \multicolumn{1}{c|}{$\begin{array}{c}\text { Anthropologische } \\
\text { Landeskunde }\end{array}$} & $\begin{array}{l}\text { Explizite, programmati- } \\
\text { sche Landeskunde }\end{array}$ & $\begin{array}{l}\text { Landesübergreifende } \\
\text { Problematisierung }\end{array}$ \\
\hline $\begin{array}{l}\text { Berücksichtigt die kulturelle } \\
\text { Dimension des sprachli- } \\
\text { chen Zeichens und reflek- } \\
\text { tiert die unterschiedlichen } \\
\text { Referenzfelder von Wörtern }\end{array}$ & $\begin{array}{l}\text { Zielt im weitesten Sinne } \\
\text { auf Verhalten und } \\
\text { Einstellungen und analy- } \\
\text { siert Kommunikations- } \\
\text { störungen }\end{array}$ & $\begin{array}{l}\text { Vermittelt Orientierungs- } \\
\text { wissen über im weitesten } \\
\text { Sinne gesellschaftliche } \\
\text { Gegebenheiten und } \\
\text { Entwicklungslinien }\end{array}$ & $\begin{array}{l}\text { Beschäftigt sich vor allem } \\
\text { mit globalen Entwicklungen } \\
\text { wie Umweltzerstörung, } \\
\text { modernen Technologien, } \\
\text { Hunger, Drogen usw. }\end{array}$ \\
\hline
\end{tabular}

Diese Einteilung in semantische, wissensbezogene und verhaltenszentrierte Landeskunde ("usos \& costumbres") ist auch in der Fremdsprachendidaktik anderer Sprachen zu finden, wobei sich in den letzten Jahren der weite Begriff "cultural awareness" entwickelt hat, der bei Penning ungefähr die Bereiche "sprachbezogene, implizite" und "anthropologische Landeskunde" umfasst.?

Diese Beispiele aus der Theorie rund um das Fach Landeskunde sollen hier nur andeuten, wie weit die Vorgabe des spanischen Erziehungsministeriums für germanistische Studien von modernen Landeskundekonzepten abweicht und in keiner der bisherigen Bildungsreformen revidiert wurde ${ }^{8}$ : im Mittelpunkt stehen Wissensinhalte und "Bildungswerte" der fremden Sprache und Kultur, wie historische Daten, Betrachtungen fremder Kulturmerkmale, berühmte Künstlerlnnen etc., also einem bildungsbürgerlichen Modell von „Kulturkunde" der 20er Jahre, das z.B. der Bildung von Stereotypen Vorschub leistet. ${ }^{9}$

Zwischen September 2001 und Oktober 2002 hatte ich die Gelegenheit, an insgesamt 12 spanischen Universitäten eine Befragung unter Studierenden der Germanistik, Übersetzungs- und Dolmetscherwissenschaft und der deutschen Sprache durchzuführen . Der Befragungsbogen von insgesamt 14 Fragen war ursprünglich von Prof. Dr. Jesus Hernandez Rojo, Universidad de Salamanca, und mir, damals DAAD-Lektorin an der Universitat de Barcelona, entworfen worden. Ziel und Absicht der Umfrage war, weitere Informationen über die Landeskunde-Lehre an spanischen Universitäten zu sammeln und damit zu einer Belebung landeskundlicher und interkultureller Reflexionen anzuregen".
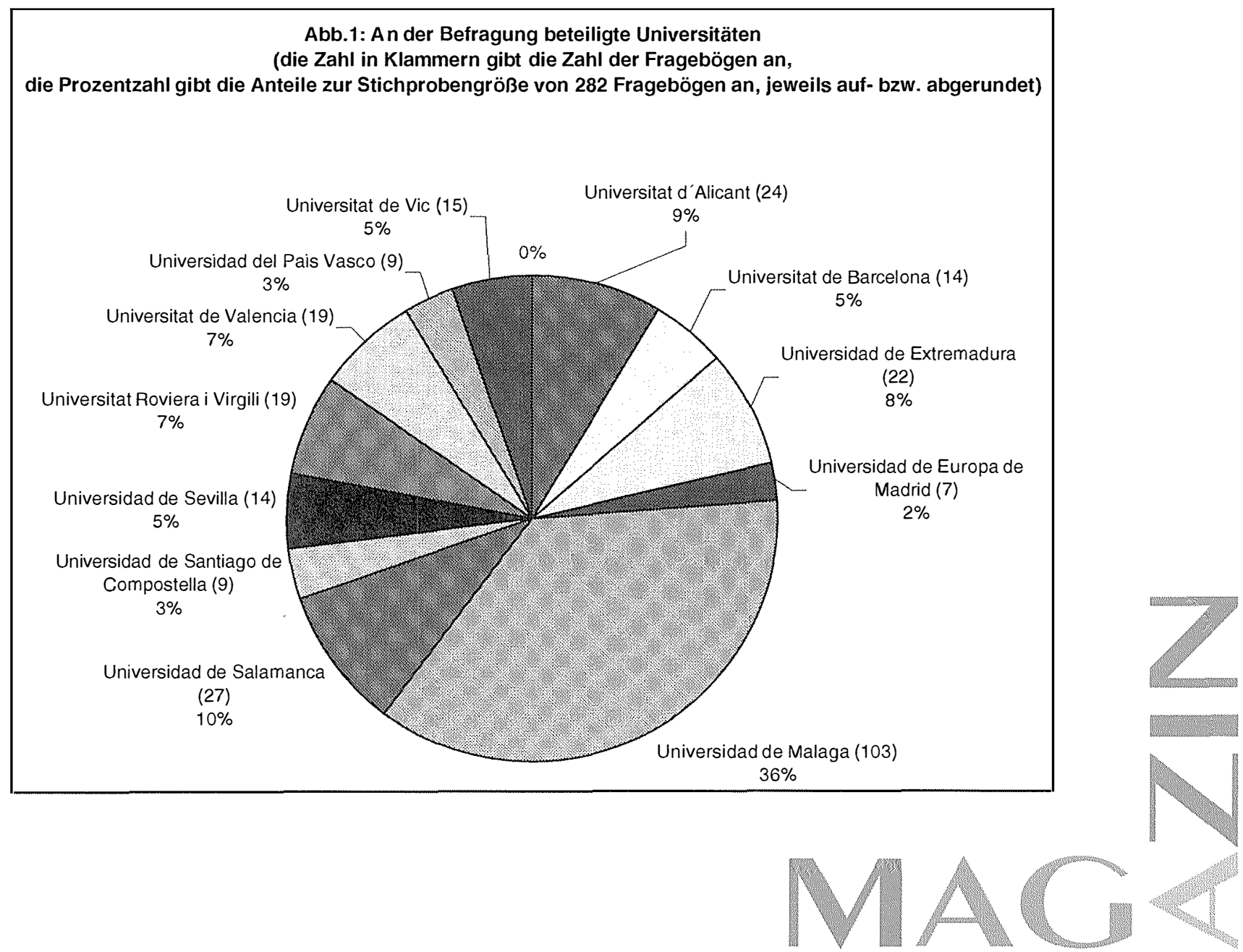
Mich interessierte bei der Befragung auf inhaltlicher Ebene, ob sich das Interesse der Deutsch-LernerInnen sich tatsächlich so stark auf Geschichtswissen konzentriert, wie in den wenigen, spanischsprachigen Lehrmaterialien zum Landeskundeunterricht vorausgesetzt wir $\mathrm{d}^{12}$. Auf methodischer Ebene interessierte mich, ob das kognitive Lernmodell des "klassischen LK-Konzepts" den Interessen und Erwartungen der Studierenden entspricht. ${ }^{13}$

Der Rücklauf ${ }^{14}$ - insgesamt 282 Studentinnen und Studenten - war umfassend genug um hiermit eine erste repräsentative Erhebung über die Beurteilung des Landeskundeunterrichts an spanischen Universitäten durch Studierende vorzulegen. (s Anhang 1). Dies ist von besonderer Bedeutung, da landeskundliche Themen oder Methodikansätze selten auf nationalen Germanistik/ DaFTagungen zu finden sind und meines Wissens nach kaum Austausch unter den Lehrenden landeskundlicher Seminare an spanischen Universitäten stattfindet ${ }^{15}$.
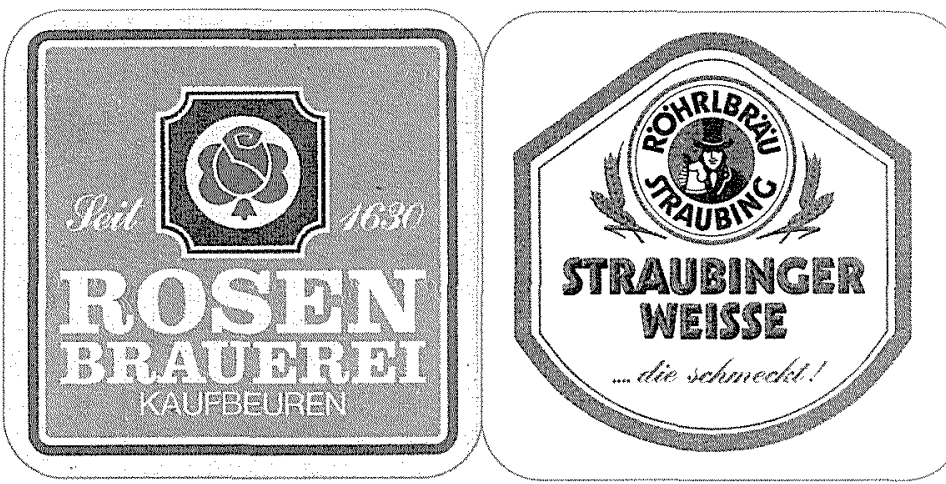

Im Zentrum des Interesses stand also die Befragung der studentischen Bedürfnisse bezüglich des Faches "cultura y sociedad" oder "Civilización Alemana" o.ä., das in die deutsche Sprache übersetzt "Landeskunde" genannt werden müsste, dabei aber seine inhaltliche Aussage und Konnotation verändert ${ }^{16}$.

\section{Die Ergebnisse der Befragung}

Frage 1: ¿Qué estudias?

Frage 2 : ¿En que curso estás? ¿Cuánto tiempo llevas estudiando alemán?

Frage 3: La asignatura de Cultura Alemana ("Deutsche Landeskunde") para ti constituye un curso: troncal; obligatorio; libre elección; ...

Bei der Beantwortung dieser Fragen zeigte sich, dass eine noch größere Vielfalt an Rahmenbedingungen für den
Landeskundeunterricht in Spanien existiert als für die Deutschstudien. Die hier vorgelegte Befragung bezieht sich auf eine Studentengruppe mehrerer (geisteswissenschaftlicher) Studiengänge, z.T. auch (ehemaliger) StudentInnen naturwissenschaftlicher Fächer. Insgesamt nahmen 163 StudentInnen verschiedener Übersetzer- und Dolmetscherfakultäten Spaniens an der Umfrage teil, sowie 66 GermanistikstudentInnen. Diese beiden Hauptgruppen werden ergänzt durch 16 Studierende der Hispanistik (Universidad de Extremadura), 5 Studierende der Englischen Philologie (Universidad de Malaga \& Universitat de Valencia), und 5 Studierende des Fachs Geschichte (Universidad de Extremadura), sowie 14 Studierende eines deutschen Sprachkurses am Spracheninstitut (Instituto de Idiomas) der Universidad de Sevilla. Vielfach wurde von den Befragten auch angegeben, dass sie neben dem Studium an Sprachkursen in Escuelas Oficiales de Idiomas oder Privatkursen teilnahmen.

Die Mehrheit der Studierenden belegten die evaluierten
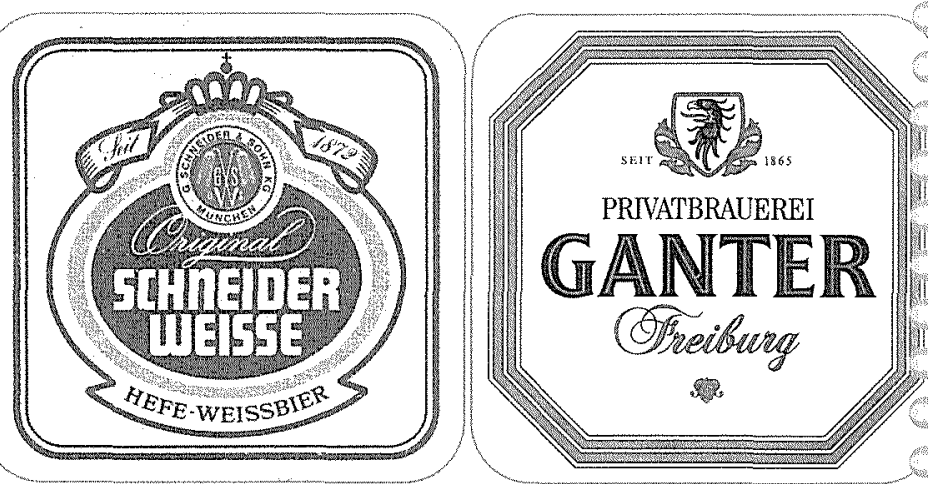

Landeskundeseminare ab dem 2. Studienjahr (durchschnittliche Studienzeit 5 Jahre), tendenziell aber mehr gegen Ende des Studiums. Auffällig war auch, dass sowohl in den Germanistik- als auch Übersetzer-/ Dolmetschenstudiengängen die meisten StudentInnen das Studium der deutschen Sprache und Kultur mit sprachlich 0-Vorkenntnissen aufgenommen hatten.

Alle gegebenen Antworten auf Frage 3 zusammengefasst ergaben, dass die Mehrheit der Befragten Landeskundeunterricht als "obligatorio" (Pflichtfach) mit 88 Nennungen belegten, wobei hier die starke Vertretung der Studienordnung Malagas eine wichtige Rolle spielt. Fast gleichgewichtig wurde "libre elección" angegeben (85 Nennungen), an 3. Stelle stand "troncal" (Pflichtfach für dieses Studienfach an allen Universitäten Spaniens) mit 68 Nennungen. Die Definition „optativa” (Wahlfach unter einer Reihe von Fächern innerhalb derselben Fakultät) wurde insgesamt $17 \mathrm{Mal}$ schriftlich ergänzt. 


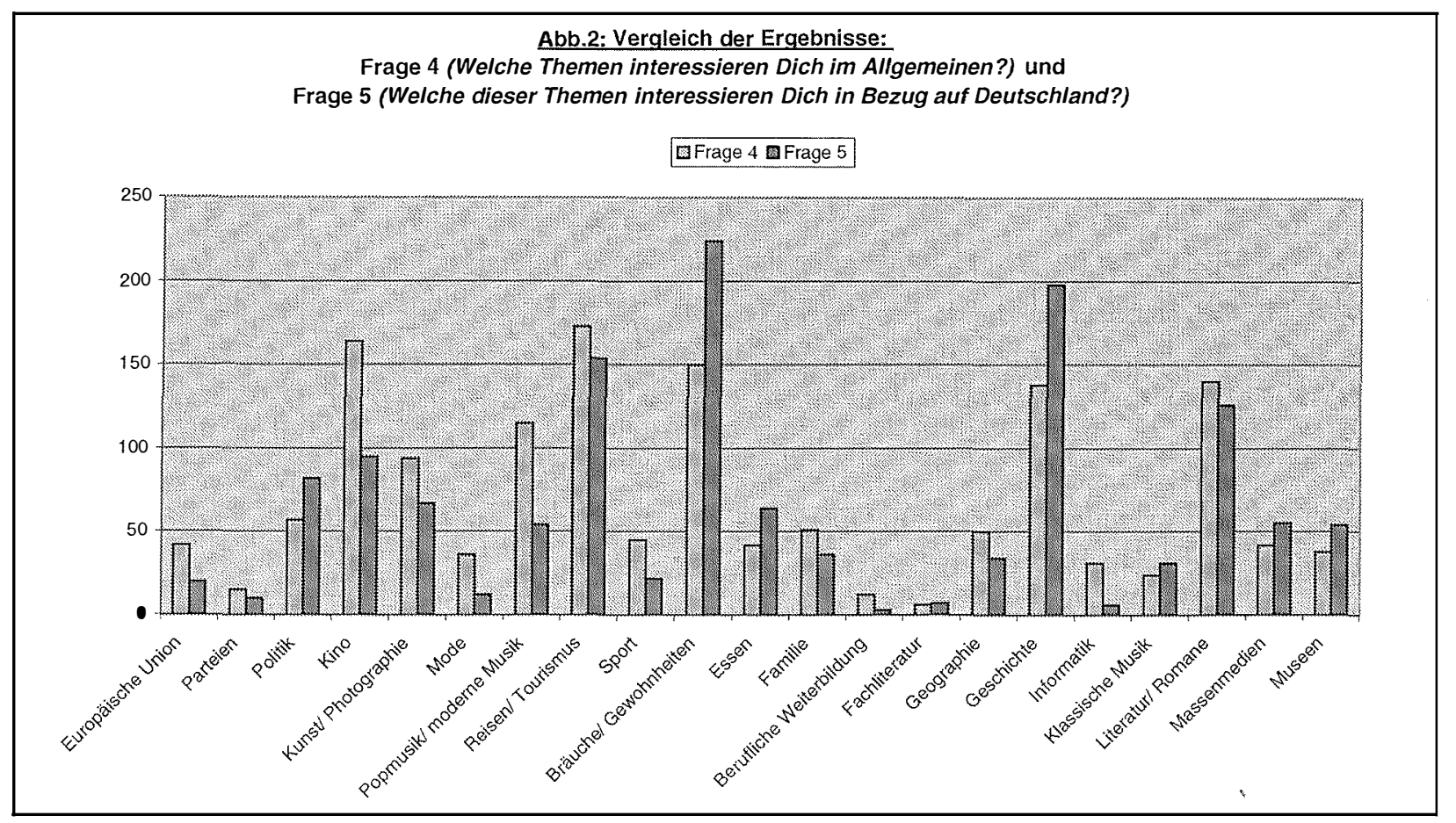

Frage 4: ¿Qué temas te interesan en general? Frage 5: ¿Cuáles de estos temas te interesan con relación a Alemania?

Mit den Fragen 4 und 5 sollte evaluiert werden, wo die Interessen der Studierenden im Allgemeinen in ihrem Herkunftsland und ihrem alltäglichen Bereich liegen. Gegeben wurde dabei eine größere Vielfalt an Antwortmöglichkeiten (23 insgesamt), die vom Umfang her überschaubar bleiben. Die Studierenden wurden auch aufgefordert, eigene ging eine Analyse mehrerer spanischer Jugendzeitschriften („Bravo“ u.a.) voraus, vermischt mit denen im Studium üblicherweise angebotenen, „klassischen" landeskundlichen Themen (Geographie, Geschichte, Politik...).

Insgesamt zeigte sich, dass sich die Interessensgebiete der Studierenden an den befragten Universitäten trotz unterschiedlicher Studienschwerpunkte, unterschiedlicher Studienordnungen und regionaler Unterschiede auf einige wenige Gebiete konzentrieren. Und dies sowohl in Bezug auf ihre eigene Kultur als auch die Fremdkultur Deutschlands.

Auffällig ist die starke Nennung der Bereiche „Bräuche/ Gewohnheiten" und "Geschichte" mit deutlicher Ausrichtung auf Deutschland. Das Interesse an Phänomenen der Alltagskultur, wie z.B. Kino, Popmusik/ moderne Musik und Kunst/ Photographie richtet sich mehr auf die eigene, spanische Kultur, wobei auch dort eindeu- tiges Interesse gezeigt wird. Das große Interesse an "Reisen" (im eigenen Land) und "Tourismus" (im fremden Land) lässt sich auf die Tatsache zurückführen, dass es sich bei den Befragten zum Großteil um Deutschstudierende bzw. DeutschlernerInnen handelt: Philologiestudien waren schon immer wegen ihrer Verbindung zum Reisen attraktiv. Wie bei den Antworten auf Frage 12 deutlich wurde, planen die meisten Studierenden einen Deutschlandaufenthalt als festen Bestandteil ihres Deutschstudiums ein.

Das deutliche Interesse an deutscher Geschichte, mehr als an der eigenen Landesgeschichte, wurde mir auch von einer Kursdozentin bestätigt, die selbst bereits Kursevaluationen durchgeführt hatte und dabei von den StudentInnen explizit um die Vermittlung historischen Wissens gebeten worden war. Das Themengebiet Geschichte gehört ebenso wie "Literatur/ Romane" zu den traditionellen Prüfungsthemen innerhalb der philologischen Studienordnungen und bestätigt ein Untersuchungsergebnis Pichts (1980) unter französischen GermanistikstudentInnen, in dem sich zeigte, dass

„die Möglichkeit zur Beschäftigung mit deutschen Themen primär nicht durch das Interesse an den deutschen Zusammenhängen geprägtist, sondern durch die Traditionen und Systemzwänge spezifischer gesellschaftlicher Situationen im jeweiligen Ausgangsland. Entscheidend ist hierbei vor allem das Prüfungssystem bzw. die Voraussetzungen und Aufstiegsmuster beruflicher Karriere."17 
D.h., dass durch die Prüfungsordnungen der jeweiligen Studiengänge auch das Interesse der StudentInnen beeinflusst und gelenkt wird. Daher die Antworten der befragten StudentInnen zu ihrem Interesse an deutscher Alltagskultur wie Pop-Musik (im Allgemeinen mit 9\%, in Bezug auf Deutschland mit 6\%), "Kunst/Photographie" (bei Frage 4 sowie Frage 5 jeweils 5\%) und "Essen" (auch gleichbleibend bei beiden Fragen von 6\% angegeben). Klassische Landeskundebereiche wie "klassische Musik", "Museen" oder "Parteien" (nach dem Vorbild der "lenguas clásicas") erhielten von den Studierenden eine glatte Absage.

\section{Frage 6: ¿Has tenido/ tienes contactos personales o familiares con Alemania?}

Mit dieser Frage sollten die persönlichen Ausgangsbedingungen der Deutschlandinteressen und informationen unter den Studierenden erfasst werden, da ja oft erst individuelle Erlebnisse (Sprachlernbiographien) die Voraussetzungen für späteres Interesse an einem
Sprachstudium schaffen. Von dem Angebot, die vorgeschlagenen Antwortmöglichkeiten zu ergänzen, wurde von Seiten der Studierenden bei der Befragung so zahlreich Gebrauch gemacht, dass es bei der Auswertung zu zahlreichen Widersprüchen bzw. nicht entschlüsselbaren Ergänzungen einzelner Antworten kam. Aus diesem Grund musste Frage 6 aus der Gesamtauswertung herausgenommen werden. Hier liegen noch viele offene Fragen für die DaF-/ Sprach-Forschung in Spanien, die besser mit qualitativen Befragungsmethoden untersucht werden sollten.

\section{Frage 7: ¿Recuerdas en clase de lengua o en la uni- versidad haber pensado que te gustaría saber más sobre algún tema en concreto? ¿Recuerdas a qué ámbito pertenecía este tema?}

Die Frage 7 stellt eine Ergänzung bzw. Gegenprobe zu den Fragen 4 und 5 dar, wobei den Befragten eine Situation geschildert wird, durch die sie aufgefordert werden, ihren Wissensbedarf bzw. ihre Interessen selbst in größere Themengebiete einzuordnen („Erinnerst Du Dich ...").

Abb.3: Frage 7: Erinnerst Du Dich, ob Du im Sprachunterricht oder an der Uni schon einmal gedacht hast, dass Du gerne mehr über ein bestimmtet Thema wissen möchtest? Erinnerst Du Dich, zu welchem Bereich dieses Thema gehörte?

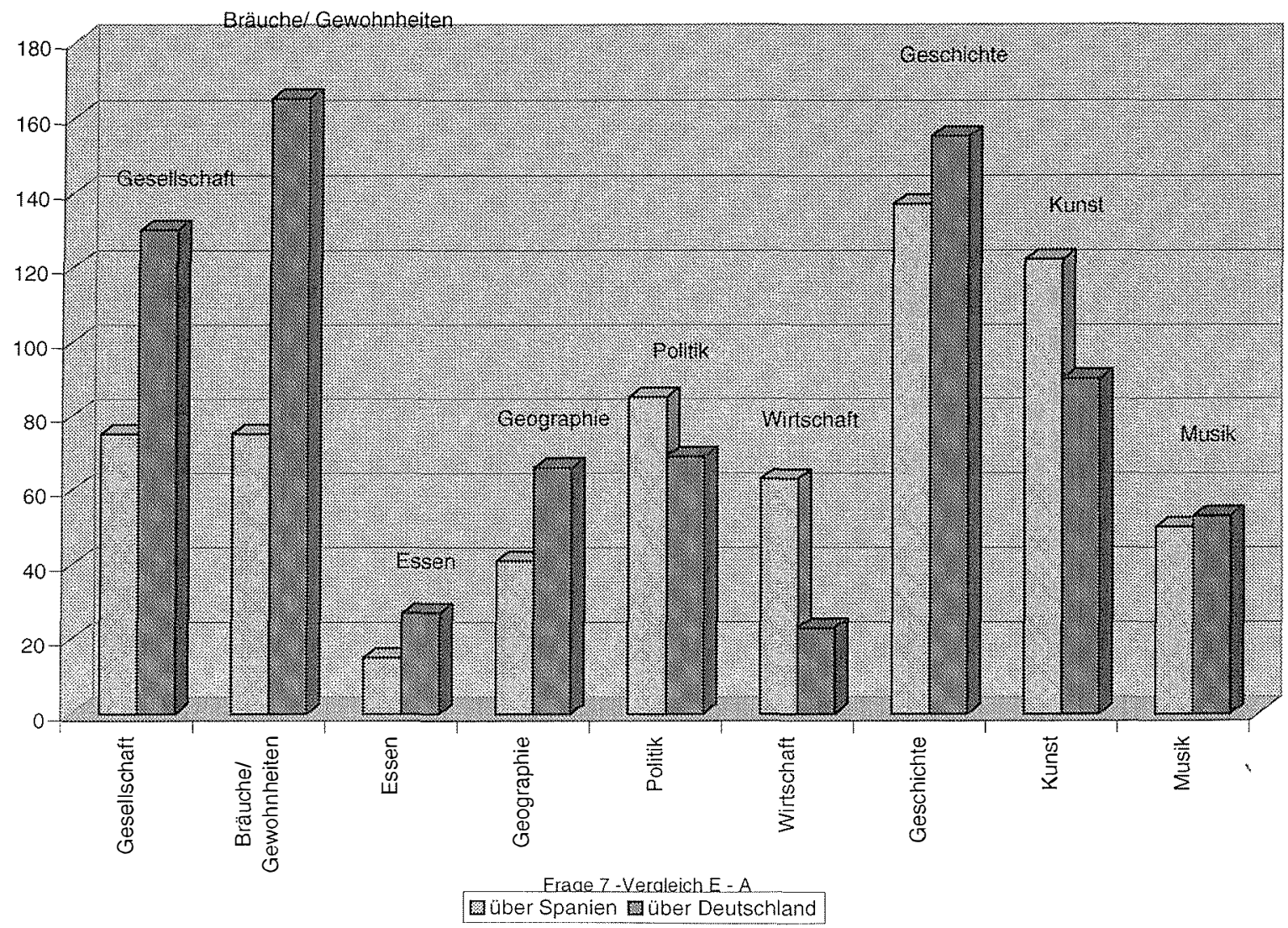




\begin{tabular}{|c|c|c|c|c|}
\hline $\begin{array}{c}\text { GenanNTE } \\
\text { TheMENBEREICHE }\end{array}$ & $\begin{array}{c}\text { ÜBER SPANIEN } \\
\text { ABSOLUTE ZAHLEN } \\
\text { VON INSGESAMt } 664 \\
\text { NENNUNGEN ( } 282 \\
\text { BEFRAGTE) }\end{array}$ & Prozentangaben & $\begin{array}{c}\text { ÜBER DEUTSCHLAND } \\
\text { ABSOLUTE ZAHLEN } \\
\text { VON INSGESAMt } 779 \\
\text { NENNUNGEN } \\
\text { (282 BEFRAGTE) }\end{array}$ & Prozentangaben \\
\hline GeselLschaft & 75 & $11,2 \%$ & 130 & $16,6 \%$ \\
\hline BRÄUCHE/ GEWOHNHEITEN & 75 & $11,2 \%$ & 165 & $21,1 \%$ \\
\hline ESSEN & 15 & $2,2 \%$ & 27 & $3,4 \%$ \\
\hline GEOGRAPHIE & 41 & $6,1 \%$ & 66 & $8,4 \%$ \\
\hline POLITIK & 85 & $12,8 \%$ & 69 & $8,8 \%$ \\
\hline WIRTSCHAFT & 63 & $9,4 \%$ & 23 & $2,9 \%$ \\
\hline GESCHICHTE & 137 & $20,6 \%$ & 155 & 19,8 \\
\hline KUNST & 122 & $18,3 \%$ & 90 & $11,5 \%$ \\
\hline Musık & 50 & $7,5 \%$ & 53 & $6,8 \%$ \\
\hline
\end{tabular}

Diese Ergebnisse bestätigen und konkretisieren die Umfrageergebnisse aus Frage 4 und 5: Die Studierenden haben im Sprachunterricht offensichtlich die Erfahrung gemacht, dass ihnen auch bezüglich ihrer Heimatkultur Wissen fehlte, insbesondere Fachwissen wie im Bereich Kunst (18,3\% der Gesamtnennungen), Politik (12,8 \% der Nennungen), und Wirtschaft $(9,4 \%$ der angekreuzten Themenbereiche über Spanien). In allen anderen Wissensgebieten vermissten die Befragten in erster Linie Kenntnisse über die Fremdkultur Deutschland, dabei mit großem Abstand wieder im alltagskulturellen Bereich der "Bräuche und Gewohnheiten"( bezüglich Deutschlands: $21,1 \%$, bezüglich Spaniens nur die Hälfte mit $11,2 \%$ ) und in dem Themenbereich, der bei dieser Frage mit dem Begriff "Gesellschaft" umfasst wurde (über Deutschland 16,6 \% und über Spanien 11,2 \%). Die Bezeichnung "Gesellschaft" wurde hier gewählt, um - in Abgrenzung zu Themengebieten wie "Politik" oder "Wirtschaft" oder "Geographie" - das gesellschaftliche Leben in seinen unterschiedlichen Facetten wie Familie, Religion, Alltagsorganisation, Wohnen, Erziehungssystem etc. zu erfassen. Dass dieses mit im Mittelpunkt des Interesses bzw. notwendigen Kulturwissens der Fremdsprachenlernerlnnen steht, beweist die hohe Nennung.

Wie bei Frage 4 und 5 steht dem alltagkulturellen Wissensbedürfnis der befragten Studierenden ein hoher Wissensbedarf in den "klassischen" Bildungsfächern Geschichte und Kunst gegenüber, wobei das Interesse an der eigenen Kultur hier nur unwesentlich geringer ist als an derFremdkultur Deutschland. (Wissenslücken wurden bezüglich deutscher Geschichte von 19,8 \% der Befragten empfunden, bezüglich der spanischen Geschichte von 20,6 \%). Auffällig ist auch wieder der geringe Wissensbedarf im Bereich "Musik" (mit 7,5 \% liegt das vermisste Wissen in der Herkunftskultur höher als in der Fremdkultur $(6,8 \%)$ (auch hier täuscht die Graphik), das gleiche Verhältnis finden wir bezüglich dem vermissten Wissen über Kunst (18,3\% über spanische und nur 11,5\% über deutsche Kunst!).

Hier wird seitens der Studierenden das klassische Bildungsverständnis in Bezug auf die eigene Kultur bestätigt, bezüglich der Fremdkultur aber abgelehnt. Was den Befragten bisher an kognitivem Wissenhintergrund innerhalb ihrer Ausbildung fehlte, bezieht sich - wie schon in Frage 4 und 5 zu erkennen war - auf die gesellschaftliche und alltägliche Realität des fremden, neuen Landes. Bildungsbürgerliche Wissenslücken werden eher in Bezug auf die eigene Kultur als fehlend empfunden.

Frage 8: Para obtener información nueva sobre algún tema (sin importar cuál), ¿a qué medio de comunicación das más credibilidad (en cuanto a la fiabilidad de la información transmitida en, por ejemplo, los atentados en los EEUU)?

Hintergrund dieser Fragen sind die Erfahrungen mit den oft gelobten, „authentischen" Informationsquellen oder -texten, die in der Landeskundetheorie seit den 70er Jahren immer wieder angepriesen werden. Von Saalbach/ Wolff u.a. wurden in den letzten Jahren auf die großen Schwierigkeiten der spanischen Deutschlernerlnnen in der Textarbeit bzw. beim Leseverstehen hingewiesen, was auf die schwache sprachliche Ausbildung der Schulkinder zurückzuführen ist. In Bezug auf den Landeskundeunterricht lenkt die Diskussion um Textverständnis jedoch vom eigentlichen Problem der Wissensvermittlung ab. Denn: wenn authentische landeskundliche Texte/ Materialien (z.B. in Lehrwerken, auf

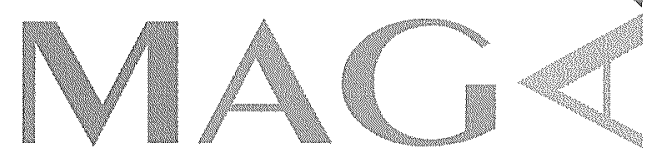




\section{THEORIE UND PRAXIS}

Videos etc.) von den LernerInnen aus sprachlichen Gründen nicht verstanden werden können, heißt das, dass man auf die Vermittlung dieser Informationen über Deutschland verzichten sollte? Tatsache ist, dass für einen kulturbewussten Sprachunterricht viel (Vor-)Wissen über das Zielsprachenland Deutschland (Österreich und die Schweiz betrifft dieses Problem noch viel mehr) fehlt und dieses daher schnell und dennoch mit Rücksicht auf den Sprachstand der Lernerlnnen in die Lehre integriert werden muss. Aus diesem Grund scheint die Frage wichtig, wie und wann (ab welchem Punkt im Sprachlernprozess) landeskundliches Wissen unterrichtet werden kann und soll, wobei hier die nach von Penning (1995) "explizite, programmatische Landeskunde" gemeint ist.

Insgesamt war bei der Auswertung dieser Fragen deutlich zu erkennen, zu welchem Zeitpunkt die Befragung an der jeweiligen Universität durchgeführt worden war: Es zeigte sich, dass die Glaubwürdigkeit aller Medien nach den Anschlägen am 11. September 2001 auf das World Trade Center in New York deutlich abgenommen hatte und großteils nur den Informationen mehrerer Medien Glauben geschenkt wurde (insgesamt 22,1\% aller gegebenen Antworten). Die Befragten in den weiter zurückliegenden Monaten schienen dagegen unbefangener mit der Informationsquelle der Massenmedien umzugehen.
Nennungen) viel Seriösität zugesprochen (insgesamt 13,7 und 9,2\% der Gesamtnennungen) ${ }^{18}$. Das Radio, das in der spanischen Medienlandschaft insgesamt keine große Bedeutung einnimmt, spielt mit $11,6 \%$ der Nennungen eine relativ große Rolle als Informationsquelle. Erstaunlich hoch schnitten auch Fachzeitschriften als mögliche Informationsquelle ab. 15,1\% der Studierenden lesen lieber eine eher populärwissenschaftlich gehaltene Fachzeitschrift als ein "wissenschaftliches Fachbuch" (7\%). Dieses Ergebnis könnte so gedeutet werden, dass der Lektüre wissenschaftlicher Fachliteratur insgesamt wenig Aufmerksamkeit geschenkt wird und viele Studierende nicht daran gewöhnt sind, sich wissenschaftlich mit Themeninhalten auseinander zu setzen.

Frage 10: En los periódicos españoles existe mucha información sobre Alemania y la política alemana. ¿Te interesan estos articulos? ¿Por qué? ¿Por qué no?

Frage 10 war die erste offene Frage innerhalb des Fragebogens und ist von den Befragten zum großen Teil recht ausführlich beantwortet worden. Die Antworten wurden in Form von Clustern in positive $(+)$ und negative (-) Aussagen eingeteilt. Von insgesamt 262 Nennungen lassen sich die Antworten in 59,4\% (absolute Zahl: 156) positive und 40,4 \% (absolute Zahl: 106) negative Stellungnahmen aufteilen, wobei sich einige Universitäten vom Tenor her maßgeblich unterschieden.

\begin{tabular}{|c|c|c|}
\hline WeLCHES ÖFFENTLICHE MedIUM? & $\begin{array}{c}\text { ABSOLUTE ZAHLEN } \\
\text { VON INSGESAMt } 582 \text { NENNUNGEN } \\
\text { (282 BEFRAGTE) }\end{array}$ & Prozentangaben \\
\hline Die ZeItUNG ... & 80 & $13,7 \%$ \\
\hline MEINE ZEITUNG, DIE ICH IMMER KAUfE & 54 & $9,2 \%$ \\
\hline EINE FACHZEITSCHRIFT ÜBER DAS THEMA & 88 & $15,1 \%$ \\
\hline EINEM BUCH VON EINER/M BEKANNTEN AUTORIN & 15 & $2,5 \%$ \\
\hline EINEM WISSENSCHAFTLICHEN FACHBUCH & 41 & $7 \%$ \\
\hline einer Statistik & 12 & $2 \%$ \\
\hline DEM TV & 92 & $15,8 \%$ \\
\hline DEM RADIO & 68 & $11,6 \%$ \\
\hline $\begin{array}{l}\text { ICH GLAUBE ES NUR, WENN DIE INFORMATIONEN BEI } \\
\text { VERSCHIEDENEN MEDIEN ÜBEREINSTIMMT. }\end{array}$ & 129 & $22,1 \%$ \\
\hline
\end{tabular}

Den Tageszeitungen, die in Spanien zumeist offen am Kiosk gekauft werden (im Gegensatz zur "Abokultur" in Deutschland) wird neben dem staatlichen (von der Regierung direkt kontrollierten) Fernsehen (15,8 \% aller
Wie einige ausgewählte Zitate zeigen, antworteten die Studierenden grundsätzlich mit Interesse am Weltgeschehen allgemein und weisen Deutschland innerhalb der Europäischen Union eine wichtige, wenn nicht die 
bedeutendste Rolle zu: "Sí, porque considero que actualmente Alemania es la primera potencia económica dentro de la U.E.." Deutsche Politik wird aber auch als Spiegel spanischer Außen- und Europapolitik genutzt: "Sí, porque me interesa la política alemana y cómo afecta su relación con la política española."

Die Mehrzahl der Befragten sind sich durch ihr Studium
Information über Deutschland gibt und wenn, dann können die gegebenen Informationen nicht eingeordnet werden: "La verdad es que no hay muchas articulos, además, aunque interesen, muchas veces no me entero muy bien porque hay muchas cosas que no sé." Besondere Kritik üben einige Befragte an der Pressefreiheit der Journalistlnnen: "No creo en la objetividad periodistica, creo más en el conocimiento histórico". Damit ließe sich

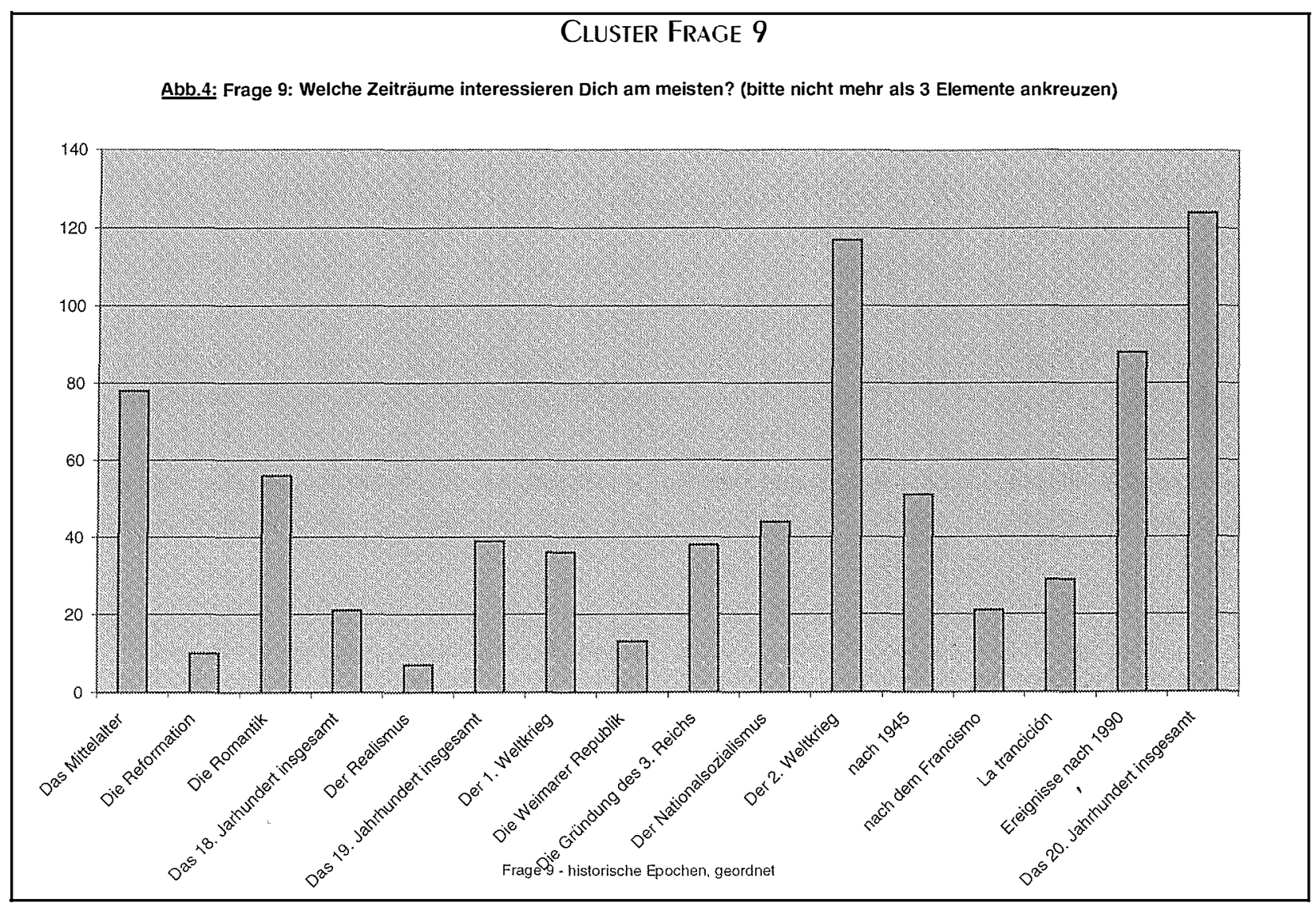

der Beziehung zwischen Sprache und Politik bewusst, auch wenn sie sich nicht explizit für das Thema Politik interessieren: "No me interesa la política" war eine häufige Antwort, aber in Bezug auf Deutschland wurde das Urteil auch wieder relativiert: "Sí, porque aunque no me interesen los temas políticos en concreto, si la sociedad en general ya que estoy estudiando el idioma."

Die Presseberichte über Deutschland selbst werden inhaltlich von den befragten Studentlnnen häufig kritisiert. Sie seien zu politiklastig und Politik und deren Presse interessieren nicht: "No suelo leer el periodico", manchmal mit der Ergänzung "No suelo leer el periodico por falta de tiempo, escucho el telediario". Kritisiert wird an den politischen Tagesnachrichten, dass es überhaupt nur wenig auch das große Gewicht erklären, das die Studierenden bei Frage 4, 5 und 7 historischem Wissen gaben.

\section{Frage 9: ¿Qué épocas históricas te interesan más?}

Frage 9 war eine Art "Spiel-" oder "Erholungsfrage". In der Reihe von insgesamt 16 Antwortalternativen wird den Befragten eine recht willkürliche Auswahl an Zeiträumen bzw. Literatur-Epochen angeboten, die einige typische Studienschwerpunkte innerhalb des Germanstikstudiums in Spanien aufgreifen.

Die Ergebnisse zeigen insgesamt, dass sich das Interesse der befragten Studentlnnen deutlich auf Ereignisse im 20. Jahrhundert konzentrieren, am deutlichsten auf den 


\section{THEORIE UND PRAXIS}

\begin{tabular}{|c|c|c|}
\hline Genannte Zeiträume & $\begin{array}{c}\text { ABSOLUTE ZaHLEN VON INSGESAMt } 772 \\
\text { NENNUNGEN (282 BeFRAGTE) }\end{array}$ & $\begin{array}{c}\text { Prozentang } \\
\text { aben }\end{array}$ \\
\hline $\begin{array}{l}\text { DAS MITTELALTER } \\
\text { DIE REFORMATION } \\
\text { DIE ROMANTIK } \\
\text { DAS 18. JAHRHUNDERT INSGESAMT } \\
\text { REALISMUS } \\
\text { DAS 19. JAHRHUNDERT INSGESAMT } \\
\text { DER 1. WELTKRIEG } \\
\text { DIE WEIMARER REPUBLIK } \\
\text { DIE GRÜNDUNG DES 3. REICHS } \\
\text { DER NATIONALSOZIALISMUS } \\
\text { DER 2. WELTKRIEG } \\
\text { NACH 1945 } \\
\text { NACH DEM FRANCISMO } \\
\text { LA TRANSICION } \\
\text { EREIGNISSE NACH } 1990 \\
\text { DAS 20. JAHRHUNDERT INSGESAMT }\end{array}$ & $\begin{array}{r}78 \\
10 \\
56 \\
21 \\
7 \\
39 \\
36 \\
13 \\
38 \\
44 \\
117 \\
51 \\
21 \\
29 \\
88 \\
124\end{array}$ & $\begin{array}{r}10,1 \% \\
1,2 \% \\
7,2 \% \\
2,7 \% \\
0,9 \% \\
5,0 \% \\
4,6 \% \\
1,6 \% \\
4,9 \% \\
5,6 \% \\
15,1 \% \\
6,6 \% \\
2,7 \% \\
3,7 \% \\
11,3 \% \\
16 \%\end{array}$ \\
\hline
\end{tabular}

Zeitraum des 2.Weltkriegs und die Vereinigung $1990^{19}$. Gleichzeitig ist erkennbar, dass die befragten StudentInnen auch den traditionellen Themen im Landeskundeunterricht folgen, wie z.B. das Mittelalter oder der Romantik, und somit auch die Einschätzung Pichts (1980) bestätigen. Bei den Studierenden scheinen zwei Interessenskategorien nebeneinander zu existieren: einerseits ist eindeutig der Gegenwartsbezug im Studium relevant, andererseits will man die traditionellen Wissensgebiete nicht ganz vernachlässigen.

\section{Frage 11: Si tuvieras que escoger un tema sobre Alemania, ¿cómo te gustaría estudiarlo?}

Unterteilt man die in Frage 11 gegebenen Antworten in Arbeitsformen unterschiedlichen Typs, lassen sich 2 Gruppierungen bilden:

- Typ A, der auf frontale Unterrichtsgestaltung und ausschließlich kognitve Wissensvermittlung ausgerichtet ist.

- Typ B, bei dem autonomes und selbstverantwortliches Lernen und Forschen Grundlage des Wissenserwerbs ist.

Bei dieser Frage zeigen die befragten StudentInnen, dass sie sich sehr selbstbewusst eigenverantwortliche Lernformen zutrauen, aber methodisch am liebsten zweigleisig fahren: Die Mehrzahl der Nennungen liegt bei der bekannten "Seminar"form, die methodisch sowohl frontal als auch mit Gruppenarbeitsphasen etc. stattfinden kann.
Frage 12: ¿Consideras que hay algunos temas sobre los que sólo se puede aprender en Alemania? ¿Cuáles?

Frage 12 war die zweite offengestellte Frage innerhalb des Fragebogens. Ausgangsinteresse war, wieviel Autorität die Studierenden dem heimischen Landeskundeunterricht überhaupt zusprechen bzw. was sie von diesem erwarten - im Gegensatz zu den Informationen, die sie im Zielsprachenland erhalten können - oder hoffen zu erhalten. Wie aus einigen Anmerkungen der Befragten hervorgeht, hat der Landeskundeunterricht für viele Fremdsprachenlernerlnnen an spanischen Universitäten überhaupt nur die Funktion, auf spätere Auslandsaufenthalte vorzubereiten. Daher sollte mit Frage 12 erfragt werden, welche Bereiche landeskundlicher Lehre die Studierenden in ihrer Erwartungshaltung aus dem universitären Lehrcurriculum ausklammern und welche authentischen Erfahrungen sie in Deutschland erwarten bzw. für sie Relevanz besitzen.

Das breite Spektrum an Antworten ergab jedoch ein ganz anderes Bild: die überwiegende Mehrzahl der Studierenden antwortete mit bestimmten, eigenen Nennungen, die ihre Erwartungen an kulturkonstrastive Erfahrungen (nicht nur in Deutschland) ausdrücken. Durch die offene Fragestellung wurde erreicht, dass die Befragten ihre Wünsche bzw. Erwartungen bezüglich landeskundlicher Erfahrungen in Deutschland in eigene 
Worte fassten, wobei sich neben den bisher im Fragebogen gebrauchten "üblichen" Begriffen, wie z.B. "usos y costumbres", zahlreiche individuelle Bezeichnungen fanden. Zusammengenommen lesen sich diese wie ein spanischer Vorschlag eines LandeskundeCurriculums für den Bereich "culture awareness".

Auffällig ist, wie klar und deutlich die Studentlnnen ihr Bedürfnis nach "lebendiger" Sprachkompetenz formulieren und aus dieser ihre Verbindung zur authentischen Kultur herstellen. Dies belegt folgendes Zitat: "Y $Y_{O}$ creo que para aprender cualquier cosa sobre un país extranjero hay que aprenderlo en él, pero hay que dominar su idioma." Einige Kommentare der Studentlnnen geben Auskunft über Erwartungen und Einschätzungen ihrer interkulturellen Erfahrungen: "Si, en mi opinión, el caracter de un pueblo sólo se puede conocer conviviendo en ese pueblo,. en este caso el alemán. "Dabei wird auch die zu kurze Lernzeit für Fremdsprachen kritisiert: „Creo que para tener una idea amplia, se necesita más tiempo, sea en España o en Alemania." Einige Befragte verneinten allerdings auch die Frage, ob man gewisse Kulturbereiche nur in der authentischen Situtation (kennen)lernen könne: „En mi opinión para lo único que es necesario estar en Alemania para aprender algo es el idioma ya que hoy la información esta en todos los lados." Eine andere Meinung war: "No, con más o menos esfuerzo se puede aprender en otros sitios aunque en algunos casos alli tendriamos más facilidades, sería más natural y espontáneo."

Frage 13: ¿ ¿Tienes la impresión de que tu interés por Alemania ha cambiado en los últimos años? Sí quieres, explica los motivos.

Frage 13 ging auf eine der Ausgangshypothesen der Umfrage zurück, bei der wir von mangelhafter Information und sprachlicher Vorbereitung vor dem Sprachstudium ausgegangen waren.

Es sollte evaluiert werden (Selbstevaluation), ob sich Verhältnis der Studierenden zur Zielkultur Deutschland im Laufe ihres Studiums verändert hat. Das Ergebnis konnte nicht klarer ausfallen: $87 \%$ beantworteten die Frage mit "Ja" und nur 13\% mit "Nein". Die Kommentare der Befragten bestätigen zudem, wie sehr Fremdsprachenunterricht erst das Interesse an fremden Kulturen weckt: „Si, cada vez me interesa más esa cultura casi desconocida para mi." und "Ha aumentado porque cuanto más me doy cuenta de lo que me falta por aprender." Auch dass Austauschprogramme, wie ERASMUSund SOKRATES große Wirkung zeigen, bestätigt sich in der Befragung: typische Kommentare waren z.B.:; "Si, desde que fui."; "Al conocer personas alemanes me interesé más por su cultura" Auch das stereotype und (teilweise) positive Deutschlandbild trägt zum positiven Ansatz mancher Studierenden bei: "Si, porque me parece un pueblo muy trabajador e interesante por su historia."

Frage 14: Si pudieras elegir, ¿dónde estudiarías Cultura Alemana (independientemente del dinero)?

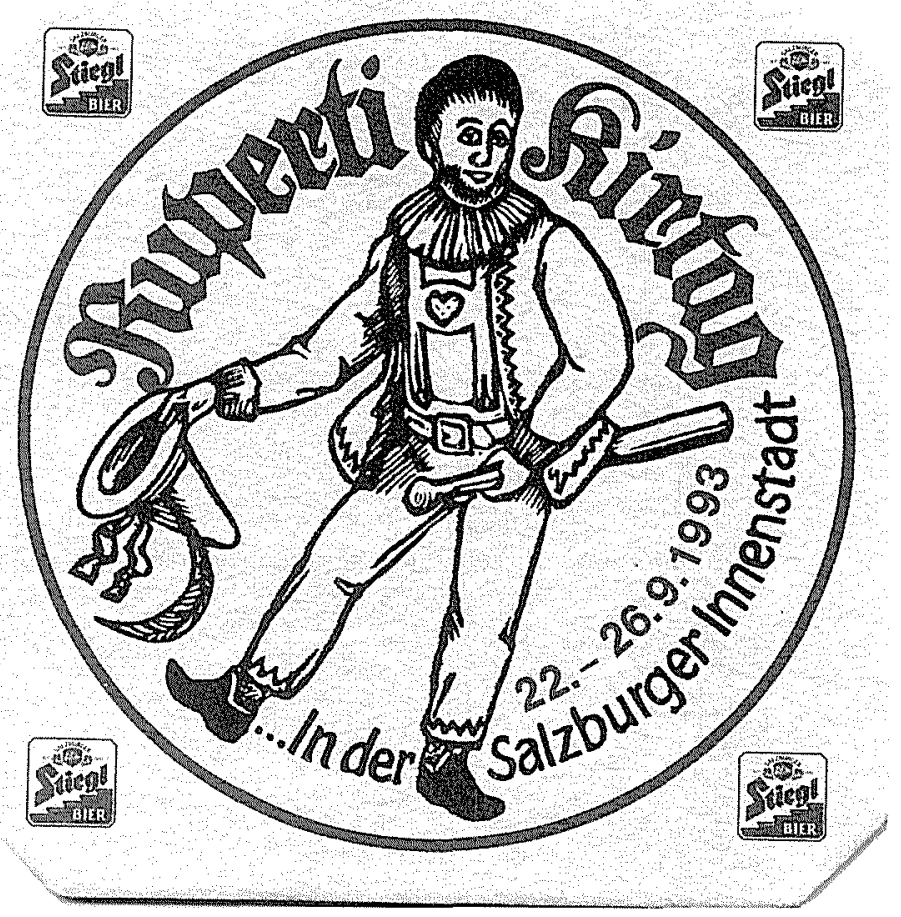

Das Ergebnis fiel äußerst eindeutig aus. Von den insgesamt 311 Nennungen sprachen sich $257(=82,7 \%)$ für einen Aufenthalt in Deutschland aus, 24 Nennungen (= $7,7, \%)$ fielen auf Österreich, 14 Nennungen $(=4,5 \%)$ auf die Schweiz, und nur $11 \mathrm{Mal}(=3,5 \%)$ wurde Spanien als bevorzugtes Studienland für Deutsche Landeskunde angegeben. Eine befragte Studentln meinte als Begründung, dass man zwar "usos y costumbres" (Bräuche und Gewohnheiten) durch Reisen im Land kennenlernen könne, "estudiar" könne man Landeskunde aber nur in Spanien. Ein anderer Kommentar gab als Grund sogar Ideologieverdacht gegenüber den nicht-spanischen Studiensystemen an: „Es una pregunta dificil ya que hay temas problemáticos que creo que en Alemania y Austria se intentan evadir por lo que sería mejor estudiarlo en España." Diese Antworten bilden aber Ausnahmen.

Nimmt man dieses Ergebnis ohne methodische Einschränkung auf, könnte es auch als harte Absage an den modernen Landeskundeansatz DACH bzw. DACHL

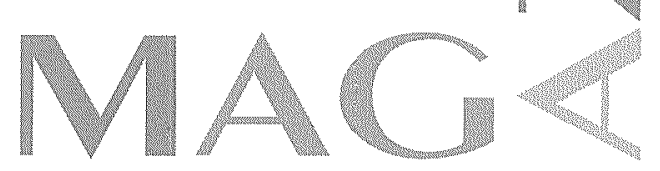


interpretiert werden. Zumindest scheint dieser im Bewusstsein der Studierenden an spanischen Universitäten in Bezug auf deutsche Landeskunde keine Rolle zu spielen - trotz zahlreicher Austausch- und langjähriger Stipendienprogramme mit österreichischen Universitäten. Auffällig an den Ergebnissen war, dass scheinbar die nationale Herkunft der an den jeweiligen Universitäten tätigen Dozentinnen Einfluss auf die Studieninteressen der Befragten hatten.

\section{Zusammenfassung}

Die Ergebnisse der Umfrage belegen eindeutig, dass die Studierenden an spanischen Universitäten ihre

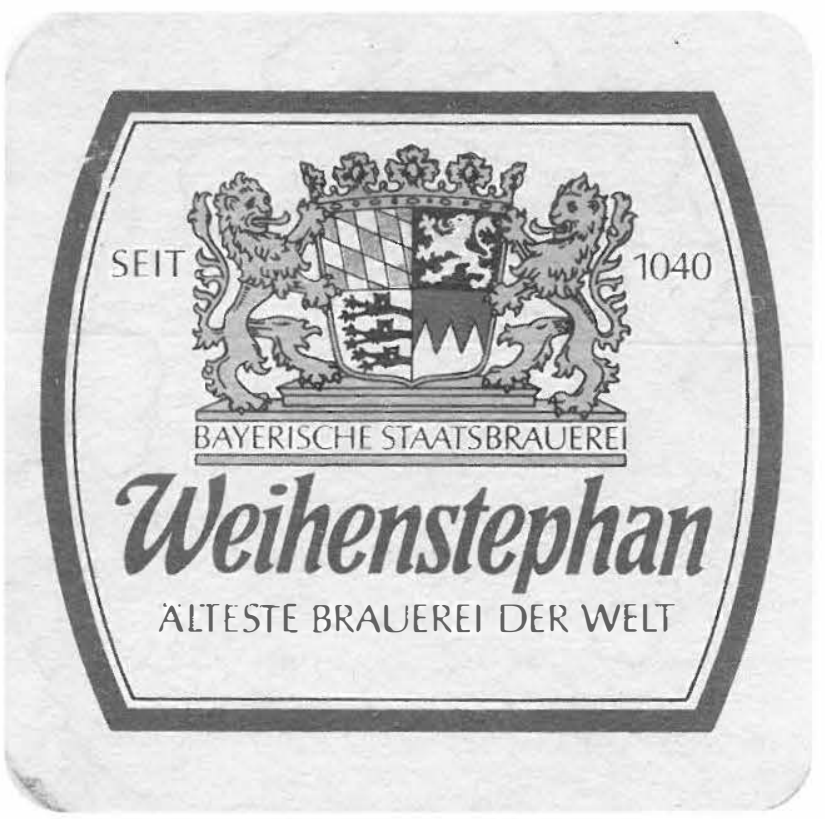

Studieninhalte bewusst wahrnehmen und reflektieren - und auch sehr genau wissen, was sie sich wünschen. Sie fordern neben dem traditionellen Wissenserwerb mehr Unterstützung durch ihr Studium für den Prozess des (Kennen-) Lernens im praktischen, realitätsbezogenen, alltagsprachlichen Kulturbereich (cultural awarness). Durch die Sprachstudien wollen sie nicht ihre eigene Persönlichkeit "bilden", sondern ins Ausland reisen und dort persönlichen Kontakt zur fremden Kultur erfahren. Gutmann (2001) fasst dies auf der Sprachebene wie folgt zusammen:

"Das Sprachwissen, das unsere Schüler haben, muss also ergänzt werden durch das Wissen, wie man Sprachen für unterschiedliche Kommunikationszwecke benutzt
(Kommuni-kationsstrategien) und durch das Wissen, wie man Sprachen lernt (Lernstrategien)."

Die befragten StudentInnen gehen sehr selbstbewusst mit autonomen Lernstrategien um, zeigen aber auch die typische "Konsumhaltung" der passiven Lernformen im traditionellen (Frontal-) Unterricht. Ihr Misstrauen gegenüber den Informationen der spanischen Presse kann nicht übersehen werden und führt den Blick zurück auf deutsche, authentische Texte/ Materialien für den Landeskundeunterricht. - Oder werfen zum wiederholten Mal die Frage nach spanischen Informationstexten im DaF-Unterricht auf. Denn es ist nicht zu übersehen, dass historischem Wissen seitens der Studierenden nach wie vor viel Gewicht gegeben wird und sie nach politisch unbeeinflussten Informationsquellen über das Zielsprachenland Deutschland suchen.

Ich glaube, die hier vorgelegten Umfragergebnisse machen deutlich, dass neue Methoden im Landeskundeunterricht mit deutlicher Betonung der Wissensvermittlung auf der Gegenwart für die universitäre Lehre ansteht.

Wie neuere Publikationen zeigen, gibt es dafür bereits einige, teilweise hervorragende, kontrastive Vorschläge innerhalb der spanischen Germanistik und Übersetzungswissenschaft (siehe Literaturverzeichnis).

\section{AnHANg 1 \\ Universitat Barcelona \\ Dpto. de Filología Anglesa i Alemanya \\ Secció d'Alemany \\ Prof.: Elisabeth F. Basteck, lectora del DAAD}

Cuestionario sobre la situación de la asignatura de Cultura Alemana en las universidades españolas.

Apreciado estudiante,

Con esta encuesta se pretende evaluar el interés de los estudiantes por los distintos aspectos de la cultura aiemana. Por favor, responde a este cuestionario de manera espontánea según tus intereses personales. Nos gustaria saber qué es lo que te interesa de Alemania o qué áreas de conocimiento echabas/ echas de menos en las clases anteriores. 
?n, wie

isst mit ie typi7 tradienüber it überutsche, den rholten ten im १, dass ch wie olitisch das

bnisse n im ig der ərsitäre

its einia innerigswis-

ura de añolas.

de los ıra alemanera zustaría áreas las cla-

\section{1.)¿Que estudias?}

Filologia alemana $\square$

Traducción e interpretación $\square$

Estoy apuntado a un curso de lengua de la escuela

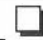

otra filologia $\square$

(¿cual?)

\section{2.)¿En qué curso estás?}

¿Cuánto tiempo llevas estudiando alemán?

3.) La asignatura de Cultura Alemana (,Deutsche Landeskunde") para ti constituye un curso:

$$
\text { troncal } \square \text { obligatorio } \square \text { libre elección }
$$

\section{4.) ¿Qué temas te interesan en general? \\ (Puedes marcar un máximo de cinco elementos) Moda $\square$ \\ Música pop/ moderna \\ Música clásica $\square$ \\ Deportes $\square$ \\ Cine $\square$ \\ Historia $\square$ \\ Viajes $\square$ \\ Museos \\ Arte/ fotografia \\ Política $\square$ \\ Medios de informaciòn \\ Informàtica $\square$ \\ Familia \\ Comida \\ Usos y costumbres \\ Literatura/ novelas \\ Literatura especializada \\ Cursos de formación profesional \\ Partidos politicos \\ La Unión Europea $\square$ \\ Sindicatos $\square$ \\ Geografia \\ Otros; especificar:}

5.) ¿Cuáles de estos temas te interesan con relación a Alemania?

(Marcar también un máximo de 5)

Moda $\square$

Música pop/ moderna

Música clásica $\square$

Deportes $\square$

Cine $\square$

Historia

Turismo

Museos $\square$

Arte/ fotografia

Politica $\square$

Medios de informaciòn $\square$

Informática $\square$

Familia $\square$

Comida $\square$

Usos y costumbres

Literatura/ novelas

Literatura especializada

Cursos de formación profesional

Partidos políticos

La Unión Europea $\square$

Sindicatos

Geografia

- Otros; especificar:

6.) ¿Has tenido/ tienes contactos personales o familiares con Alemania?

(Por favor, no marcar más de 2 elementos)

- Si, parte de mi familia vive en Alemania

- Si, tengo amigos en Alemania a los que veo regularmente $\square$

- Si, estuve algunas veces en Alemania, pero he perdido el contacto con los que conocia

- Tenia un amigo/a por carta de Alemania $\square$

- No, nunca he estado en Alemania ni conozco a alemanes personalmente $\square$

- Conozco a alemanes de vista, de la calle o la playa

7.) ¿Recuerdas en clase de lengua o en la universidad haber pensado que te gusteria saber más sobre algún tema en concreto? ¿Recuerdas a qué ámbito pertenecia esta tema? (Por favor, no marcar más de 3 elementos.) 
SOBRE ESPAÑA

Politica $\square$

Geografía $\square$

Historia $\square$

Economía $\square$

Arte $\square$

Música $\square$

Sociedad $\square$

Comida $\square$

Usos y costumbres $\square$

8.) Para obtener información nueva sobre algún tema (sin importar cuál), ¿a qué medio de comunicación das más credibilidad? (en cuanto a la fiabilidad de la información transmitida en, por ejemplo, los atentados en los EE UU) (Por favor, no marcar más de 3 elementos)

\section{La televisión $\square$}

El periódico:

La radio

El periódico que compro siempre $\square$

Un libro de un/a autor/a conocido/a

Una estadística $\square$

Una revista especializada sobre el tema $\square$

Un libro científico sobre el tema $\square$

Sólo lo creo, cuando la información coincide en distintos medios de comunicación $\square$

9.) ¿Que épocas históricas te interesan más? (Por favor, no marcar más de 3 elementos)

La edad media $\square$

La reforma $\square$

El siglo XVIII en general $\square$

El romanticismo $\square$

El siglo XIX en general

El realismo $\square$

El III Reich $\square$

El siglo XX en general $\square$

La $1^{\text {a }}$ Guerra Mundial

La República de Weimar $\square$

El nacionalsocialismo

La $2^{\text {a }}$ Guerra Mundial $\square$

Después de $1945 \square$

La transición $\square$

Después del franquismo $\square$

Acontecimientos desde 1990
SOBRE ALEMANIA

Política $\square$

Geografía $\square$

Historia $\square$

Economía $\square$

Arte $\square$

Música $\square$

Sociedad $\square$

Comida $\square$

Usos y costumbres $\square$ (Por favor, no marcar más de 2 respuestas)

- En un seminario específico sobre el tema $\square$

- A través de una conferencia de un/a especialista $\square$

- Una introducción general en una asignatura (junto a otros temas) $\square$

- Reunir información para un grupo de trabajo y después compartir los conocimientos obtenidos $\square$

- Investigar el tema independientemente y hacer un trabajo escrito sobre el mismo $\square$

- Leer un libro sobre el tema $\square$

- Tomar un semestre libre y leer varios libros sobre el tema $\square$

- Buscar un/a interlocutor/a por Internet para hablar sobre el tema $\square$ $\checkmark$

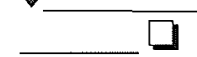

12.) ¿Consideras que hay algunos temas sobre los que sólo se puede aprender en Alemania? ¿Cuáles?

13.) ¿Tienes la impresión de que tu interés por Alemania ha cambiado en los últimos años?
Si $\square \quad$ No $\square$
Si quieres, explica los motivos:

14.) Si pudieras elegir, ¿dónde estudiarías Cultura Alemana (independientemente del dinero)?

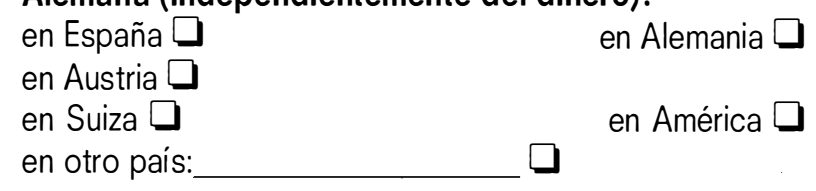




\section{Fußnoten}

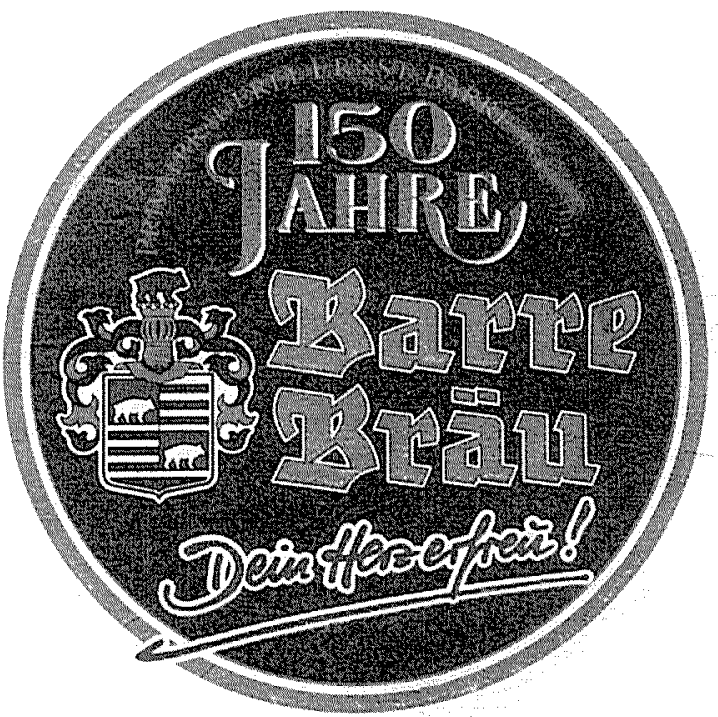

1. Siehe z.B. Torres, M./ Wolff, J. (1983), Keim (1996), u.a.

2. Laut BOE (Boletin Oficial del Estado) Nr. 278 vom 20. November 1990 mit mindestens 8 creditos.

3. Siehe dazu: Marizzi, B. (2002), "Apuntes acerca de la historia de la enseñanza del alemán como lengua con fines específicos en España", in: Goethe Institut Inter Nationes/ Instituto Alemán de Cultura, El alemán: una lengua extranjera en España, Madrid, S. 6365 und auch in: Siguan Boehmer, M. (1998), "Überlegungen zum Germanistikstudium in Spanien: am Beispiel der Universität Barcelona", in: Germanistentreffen Deutschland-Spanien-Portugal 13.-18.09.1998, DAAD (Hrsg.), Bonn: DAAD, S. 61 - 70, S.61 ff. 4. Veeck, R./ Linsmayer, L. (2001), "Geschichte und Konzepte der Landeskunde", in: Deutsch als Fremdsprache, Ein internationales Handbuch, Helbig, G./ Götze, L./ Henrici, G./ Krumm, H.-J. (Hrsg.), 2. Halbband, Berlin/ NY: de gruyter, S. 1160-1168, S. 1160 5. Dieter Penning, "Landeskunde als Thema des Deutschunterichts - fächerübergreifend und/ oder fachspezifisch?", in: Info DaF, Nr.6, 22. Jhrg. (1995), Hrsg. DAAD, Bonn, S. 626-640, S. 628 6. Dieter Penning (1995), S.628

7.Siehe z.B. Klippel (1987), in: Heuer, H./ Klippel, F., Englischmethodik, Berlin: Cornelsen, oder Pfromm, R. (1993), Einführung in die Sprachlehr- und Lernforschung, Rheinbach: CMZ-Verlag, S.237 ff., oder Byram, M./ Morgan, C. et al (1994), Teaching-and-Learningand-Culture, Cleverdan: Multilingual Matters. S. $51 \mathrm{ff}$.

8.Sehr viel fortschrittlicher lesen sich die Lehrpläne des MEC und einiger Regionalregierungen für die öffentlichen Sekundarschulen der Gesetze LGE und LOGSE, siehe dazu: Bosch Roig, G. (1999), Sprachenpolitik und Deutschunterricht in Spanien, Dissertation im Fachbereich Deutsch als Fremdsprache, Universität Bielefeld, z.Zt. im Internet publiziert unter:

http://www.goethe.de/wm/mad/pdf/DaF-SP.pdf (Gesehen: 27.2. 2003), S. 40 und $58 \mathrm{ff}$.

9. Siehe dazu die Ergebnisse der Untersuchung der Iberischen Lektorenarbeitsgruppe der DAAD-Lektorinnen und -Lektoren, "Eine Erhebung zum Deutschlandbild der Germanistikstudentinnen und -studenten auf der iberischen Halbinsel", in: Info DaF 26, 4 (1999), S. 355-377, S. 355. Sie mündet in der Forderung, "dass spezifisches landeskundliches Material erstellt werden muss, das auf Stereotypen eingeht und zur Auseinandersetzung mit ihnen anregt".

10.(In Klammern die Zahl der Befragten): Universitat d'Alicant (24), Universitat de Barcelona (14), Universidad de Extremadura (22), Universidad de Europa de Madrid (7), Universidad de Malaga (103), Universidad de Salamanca (27), Universidad de Santiago de Compostella (9), Universidad de Sevilla (14), Universitat Roviera i Virgili in Tarragona (19), Universitat de Valencia (19), Universidad del Pais Vasco in Vitoria (9), und Universitat de Vic (15).

11.Siehe dazu auch einige der Publikationen in der Literaturliste im Anhang

12. Siehe z.B. Janés, A. (1992), Literaturliste im Anhang

13. Siehe z.B. Blanco-Camblor (2002), die sich in ihrem Landeskundeentwurf nach wie vor ausschließlich auf auf eine (ungeordnete) Menge an Texten verschiedenster Art stützt, u.a. auch auf die Beschreibung von Experimenten an KZ-Häftlingen (!), S.223 ff.

14. Dr. Anton Haidl, Universidad de Cádiz, Sophie Caesar, Hueber-Verlag/ Ed.Idiomas, Madrid, meinen DAAD-Lektorenkolleglnnen sowie allen mitwirkenden DozentInnen möchte ich an dieser Stelle meinen herzlichen Dank aussprechen.

16. Leider kann ich an dieser Stelle die methodische Vorgehensweise der Umfrage nur andeuten, ausführlich sollen die Ergebnisse im Rahmen meiner Dissertation Ende 2003/ Anfang 2004 publiziert werden. („Landeskundeunterricht an spanischen Universitäten" unter Betreuung von Prof. Dr. Oomen-Welke, Pädagogische Hochschule Freiburg, und Prof. Dr. Olga Esteve, Universitat Pompeu Fabra Barcelona). Die methodische Konzeption des Fragebogens entspricht der Anwendung der quantitativen, nicht-experimentellen Forschung. Es sollten also möglichst viele vorgegebene Antworten angekreuzt und nur bei insgesamt 3 von 14 Fragen eigene Bewertungen (qualitative Forschung) gegeben bzw. individuelle Standpunkte erläutert werden. Diese stark gelenkte Befragungsmethode war notwendig, um die schriftliche Befragung überschaubar und für eine einzelne Person arbeitstechnisch in einem auswertbaren Rahmen zu halten. Bei fast allen geschlossenen Fragen wurde den Studentlnnen auch die Möglichkeit gegeben, neben den vorgegeben Antworten, eigene Punkte zu ergänzen. Dieses Angebot wurde besonders bei der Frage 6, 10 und 12 von den Studierenden häufig wargenommen.

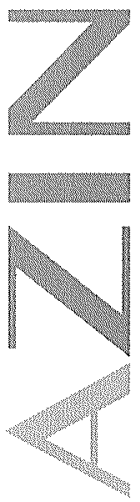




\section{THEORIE UND PRAXIS}

17. Robert Picht, "Interesse und Vergleich: zur Sozialpsychologie des Deutschlandbilds" (1980), in: Jahrbuch Deutsch als Fremdsprache 6 (1980), Hrsg. A. Wierlacher u.a., Eidelberg: Julius Verlag,S.120-132, S. 129

18. "Depende de la cadena" war einer der hinzugefügten Kommentare der befragten Studentlnnen. Weitere Studentlnnen betonen noch stärker ihre Zweifel am öffentlichen Mediensystem: „Ninguna información en los medios de comunicación es valida para mi al $100 \%$ ni aunque coincida. Todo es relativo y manipulado según el interés".

19. Ähnlich wie bei Frage 4 und 5 wirft dies die Frage auf, in wie weit Themen aus der Zeit nach dem Fall der Mauer bisher überhaupt im Curriculum des Landeskundeunterrichts an spanischen Universitäten vertreten sind - und es z.B. überhaupt ins Spanische übersetzte Quellen, Originaltexte und Informationen aus dieser Zeit gibt.

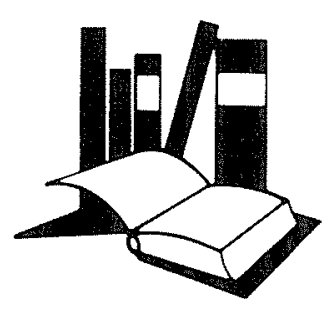

ANHANg 2

\section{Literatur zum Landeskundeunterricht in Spanien:}

- Bachmann, S. (1992), "Kontrastive Landeskunde", in: Filología Alemana y Didáctica del Aleman/ Germanistik und Deutschunterricht, Materialien des V. Symposiums des Spanischen Deutschlehrerverbandes, Valladolid 6.-10. November 1989, Regales Serna, A. (Hrsg.), S. $311-321$

- Becher, G. (1994), "Landeskunde im fremdsprachlichen Unterricht: ein integriertes Unterrichtsmodell", in: DaF aus spanischer Sicht, Primeres Jornades sobre l'Enseyament de l'Alemany en Contextos Hispánics: Perspectives Metodológiques, Barcelona: Institut de Ciéncies de l'Educació de la Universitat Autónoma de Barcelona, S. 39 - 44

- Blanco-Camblor, M.L. (2002), "Los textos literarios como transmisores de conocimientos de "Landeskunde" en clase de idiomas", in: Estudios Filológicos Alemanes, Vol.1, Universidad de Sevilla, S. 207- 225

- Decker, Th. (2001), Manual de Aleman para universitarios, Guía lingüistica para estudiar en Alemania, Austria o Suiza germanófona, Valencia: Editorial Unialemán

- Ensinger, D./ Presas, M. (1994), "Kontrastive Landeskunde in der Übersetzerausbildung. Vorschläge für die Gestaltung und Methodik des Faches "Landeskunde", in: DaF aus spanischer Sicht", Primeres Jornades sobre l'Enseyament de l'Alemany en Contextos Hispánics: Perspectives Metodológiques, Barcelona: Institut de Ciéncies de l'Educació de la Universitat Autónoma de Barcelona, S. 53 - Fulbrook, M. (1990), Historia de Alemania, Cambridge University Press, Traducción de Beatriz García Ríos (1995)

- Gutmann, A. (2001), "Neueste Tendenzen im Deutschunterricht", in: Aspectos didácticos de Alemán.1, Gutmann, A./ Arillo, M./ Hübner, D.F./ Zaera, S./ Rodríguez, J.Mª./ Jimeno, R. (Hrsg.), Zaragoza: Instituto de Ciencias de la Educación, Universidad de Zaragoza, S. 13-50 - Hammermüller, G. (1981), "Bemerkungen zu Theorie und Praxis der Landeskunde", in: Akten des 1. Iberischen Germanistentreffens, Varas, P./ Bujan Lopez, C. (Hrsg.), Salamanca: Ediciones Universidad de Salamanca, S. 211- 224

- Iberische Lektorenarbeitsgruppe der DAAD-Lektorinnen und Lektoren (1999), "Eine Erhebung zum Deutschlandbild der Germanistikstudentinnen und -studenten auf der iberischen Halbinsel", in: Info DaF, 4, Deutscher Akademischer Austauschdienst (DAAD) (Hrsg.), München: iudicium, S. 355-377

. Jané Carbó, J. (1981), "Landeskunde im Sprachunterricht", in: Akten des 1. Iberischen Germanistentreffens, Varas, P./ Bujan Lopez, C. (Hrsg.), Salamanca: Ediciones Universidad de Salamanca, S. 257-270

- Janés, Alfonsina (1992), Historia de la cultura alemana, Barcelona: MEAS S.C.

- Iglesias, F. et al. (1992), "La imagen de Alemania en Castilla-León", in: Filología Alemana y Didáctica del Aleman/ Germanistik und Deutschunterricht, Materialien des V. Symposiums des Spanischen Deutschlehrerverbandes, Valladolid 6.-10. November 1989, Regales Serna, A. (Hrsg.), S. 329-335 
- Juretschke, H. (1981), "Methodologische Randglossen zur Landeskunde in der Praxis", in: Akten des 1. Iberischen Germanistentreffens, Varas, P./ Bujan Lopez, C. (Hrsg.), Salamanca: Ediciones Universidad de Salamanca, S. 227-236

- Keim, L. (1994), "Interkulturelle Verhandlungen: der deutsch-spanische Fall", in: DaF aus spanischer Sicht, Primeres Jornades sobre IEnseyament de l'Alemany en Contextos Hispánics: Perspectives Metodológiques, Barcelona: Institut de Ciéncies de l'Educació de la Universitat Autónoma de Barcelona, S. 117 - 127

- Keim, L. (1998), "Image und Höflichkeit aus interkultureller Sichtt, in: Actas del Congreso Fundacional de la Federación de Asociaciones de Germanistas en España, Madrid: Editorial Idiomas, S.231- 244

- Kunkel, K./ Moreno, C./ Piñel, R./ Schilling, Mª L. (1995), "Das Konzept der „Frau” in den Lehrwerken für Deutsch und Spanisch als Fremdsprache", in: Fremdsprachendidaktik zwischen Tradition und Innovation/ La Enseñana de lengua extranjeras entre tradición e innovación, Raders, M./ Schilling, Mª L. (Hrsg.), Madrid: Universidad Complutense de Madrid, S. 37-70

- Laxague, A. (1992), "Objektive und subjektive Elemente in der Landeskunde. Stellenwert und Nutzen der "realen" Tatsachen", in: Filología Alemana y Didáctica del Aleman/ Germanistik und Deutschunterricht, Materialien des V. Symposiums des Spanischen Deutschlehrerverbandes, Valladolid 6.-10. November 1989, Regales Serna, A. (Hrsg.), S. 323-328

- Lüning, M. (2001), "Alemania y la Guerra Civil Española : un enfoque intercultural", in : Von der traditionellen Landeskunde zum interkulturellen Lernen, Bernecker, W.L. (Hrsg.), Berlin: edition tranvía, S. 33 - 50

- Martin de la Guardia, R. (1992), "El problema alemán después de 1945. Una aproximación a la historia reciente de Alemania", in: Filología Alemana y Didáctica del Aleman/ Germanistik und Deutschunterricht, Materialien des V. Symposiums des Spanischen Deutschlehrerverbandes, Valladolid 6.-10. November 1989, Regales Serna, A. (Hrsg.), S. 531-547

- Raders, M. (1998), "Landeskunde in der DaF-Didaktik: Überlegungen zu einem umstrittenen Fach und praktische Erfahrungen im Studiengang Germanistik an der Madrider Universität Complutense", in: Actas del Congreso Fundacional de la Federación de Asociaciones de Germanistas en España, Madrid: Editorial Idiomas, S. 245-254

- Regales, A. (1990), "Über Landeskunde in spanischen Lehrbüchern für Deutsch als Fremdsprache", in: Neusprachliche Mitteilungen aus Wissenschaft und Praxis, Fachverband Moderne Fremdsprachen (FMF) (Hrsg.), Bielefeld: Cornelsen, S. 247-253

- Regales, a. (1992), "Pacifismo y "Landeskunde"“", in: Filología Alemana y Didáctica del Aleman/ Germanistik und Deutschunterricht, Materialien des V. Symposiums des Spanischen Deutschlehrerverbandes, Valladolid 6.-10. November 1989, Regales Serna, A. (Hrsg.), S. $337-367$

- Rodiek, C. (1981), "Theorie und Curriculum der Landeskunde im spanischen Germanistikstudium," in: Akten des 1. Iberischen Germanistentreffens, Varas, P./ Bujan Lopez, C. (Hrsg.), Salamanca: Ediciones Universidad de Salamanca, S. 237-250

- Roetzer, H.G. (1994), "La enseñanza de la literatura en un contexto intercultural", in: El Aleman como lengua extranjera, Selección y revisión de Javier de Agustín, Cuadernos del tiempo libre, Colección Expolingua, Madrid: Fundación Actilibre, S. 49-55

- Saalbach, M. (1994), "Kommunikationsprobleme zwischen spanischen und deutschen Muttersprachlern aufgrund kultureller Interferenzen - Berücksichtigungsmöglichkeiten im DaF-Unterricht", in: DaF aus spanischer Sicht, Primeres Jornades sobre I'Enseyament de l'Alemany en Contextos Hispánics: Perspectives Metodológiques, Barcelona: Institut de Ciéncies de l'Educació de la Universitat Autónoma de Barcelona, S. 183 - 195

- Saalbach, M. (1999), "Kulturelle Interferenzen als Auslöser von Kommunikationsproblemen zwischen Spaniern und Deutschen - ein Problem des Landeskundeunterrichts im Bereich Deutsch als Fremdsprache und seine Perspektiven", in: Revista de Filología Alemana, 7/ 1999, Madrid, S. 309-325

- Spaniel, D. (2001), "Der Einfluss von Kommunikationswegen auf die Herausbildung von Nationen-Images: Das Deutschland-Image von Spaniern, die Deutsch lernen", Magisterarbeit. Technische Universität Dresden

- Spaniel, D. (2002), "Autos, Bier und ernste Menschen. Eine quantitaive Umfrage zum Deutschlandbild spanischer Deutschlehrer", in: mAGAzin 11, Andalusischer Germanistenverband (Hg.). S. 6-15

- Sainz Lerchundi, A./ Sánchez Hernández, J. (1997), Vocabulario de cultura alemana, Sevilla: Kronos

- Torres, M./ Wolff, J. (1983), "Interkulturelle Kommunikationsprobleme beim Sprachenlernen", in: Neusprachliche Mitteilungen aus Wissenschaft und Praxis, 36. Jhrg., S. 209-216

- Trost, K.H. (1981), "Literatur und Landeskunde", in: Akten des 1. Iberischen Germanistentreffens, Varas, P./ Bujan Lopez, C. (Hrsg.), Salamanca: Ediciones Universidad de Salamanca, S. 271 - 279

-Wessling, G., "Conocimiento intercultural del país en la enseñanza de idiomas", in: El Aleman como lengua extranjera, Selección y revisión de Javier de Agustín, Cuadernos del tiempo libre, Colección Expolingua, Madrid: Fundación Actilibre, S. 81-88

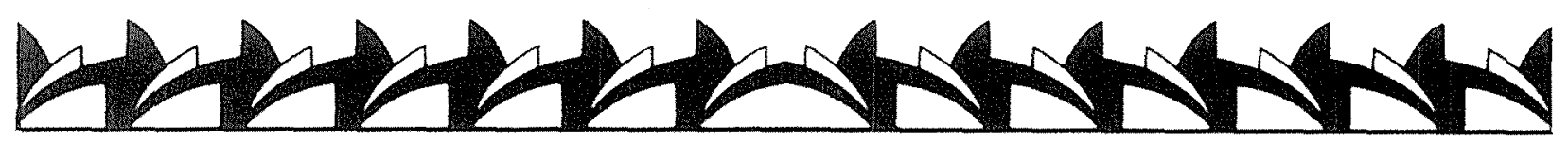

